\title{
Treating Insomnia with Shen-Related Acupoints
}

\author{
Tong Zheng Hong* \\ As-You-Wish Healthcare Institute, Taiwan
}

*Corresponding author: Tong Zheng Hong, As-You-Wish Healthcare Institute, MS in Acupuncture awarded by National University of Health Sciences in IL, Taiwan

\begin{tabular}{ll}
\hline ARTICLE INFO & ABSTRACT \\
\cline { 1 - 1 } $\begin{array}{l}\text { Received: } \\
\text { Published: December 20, } 2019\end{array} \quad \begin{array}{l}\text { In addition to HT7 which is often selected in the protocol to treat insomnia, there } \\
\text { are seven more Shen-related acupoints are presented and discussed for acupuncture } \\
\text { practitioners to consider for treating insomnia. The result in this study shows Chinese } \\
\text { names of the acupoints represent underlying functions for practitioners to follow based } \\
\text { on the severity. }\end{array}$
\end{tabular}

Keywords: Jing; Qi; Shen; Governing Vessel; Wind MS.ID.004037.

\section{Introduction}

It is estimated that one-third of adults have experienced the symptoms resulted from insomnia like falling asleep difficultly, sleep efficiency less than $85 \%$ sleep, or latency more than 30 minutes [1] while approximately $6 \%$ meet the criteria of insomnia in diagnosis, which suggests insomnia is absolutely a common sleep disorder worldwide [2]. Acupuncture is the one of the common modalities used to treat insomnia resulting in functional impairments to increase the risk of fatal health conditions like cardiovascular disease and depression in the long term, in the Chinese communities and around the world at present to restore the normal sleep-wake cycle [3]. Different from the Western medicine developed for less than three hundred years, traditional Chinese medicine (TCM) is the major healthcare system that has been developed for thousands of years. The philosophy the Western medicine follows in the development is the hypothetical deduction while Chinese medicine resides in the inductive reasoning [4], which obviously needs attention for the future development. In the TCM, the constitution of humans and patterns of the disharmony in the body are generally classified into the eight categories of YinYang, External-Internal, Cold-Hot, and Deficiency-Excess with the essential materials like Qi, Blood, and fluid accompanied by ZangFu organs differentiation [5].

\section{Three TCM Treasures}

It is understood that the human body in the TCM is seen as a microcosm of the universe and as a whole clinically in diagnosis and treatment. One can stay healthy when Yin and Yang are balanced to bring the body, mind and spirit into harmony. On the other hand, the Ying-Yang theory points out that the three treasures Jing (Essence), Qi, and Shen (Spirit) are essential for sustaining the human life in the TCM. From the aspect of Ying-Yang theory, Jing (Essence) is viewed as Ying, which refers to the essential substance and seems to have the high similarity to genes. Prenatal Jing is recognized by the ancient TCM masters that it is an important substance passed from parent to child, and may cause serious health issues in development, growth, and reproduction. Qi, the required energy of the body classified as Yang, represents functions like coordinating organs, warming the body up, transforming food into useful substances, and defending the body against external evil Qi (pathogens) as the source of all movements of the body. Normally speaking, both Jing and Qi collaborate with each other shown in Figure 1 for the manifestation of Shen. Shen, which can to some extents imply the consciousness, mental functions, mental health, and vitality [6], can serve as the key tool for diagnosis with pattern identification derived from the Ying-Yang theory, which 
distinguishes TCM from the Western medicine and indicates that a disease represents the loss of the balance between Yin and Yang [7].

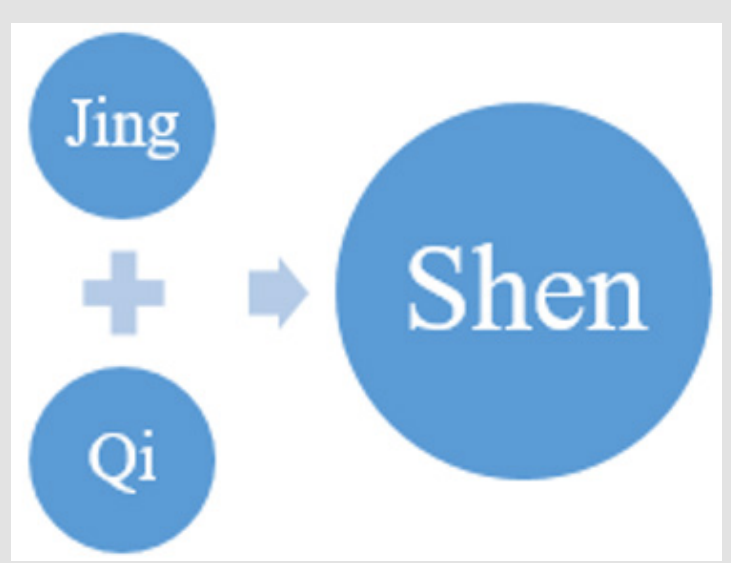

Figure 1: Relationship of Jing and Qi with Shen.

\section{Pathological View of Insomnia}

Insomnia is understood to be the loss of the Yin-Yang balance [8], indicating the fighting between Yin and Yang. The most important key to the successful treatment outcomes requires the experi- enced TCM practitioners and acupuncturists to rely on the pattern identification for the accurate diagnosis to give the prescriptions with the four skills- looking, smelling, questioning, and pulse taking. In other words, pattern, which can surely distinguish the TCM from the Western medicine, should be the key concern for the TCM and acupuncture practitioners in the decision-making of treatments. The positive and correct identification of patterns developed with the Yin-Yang theory must be strictly followed for the expected outcomes since a disease is the presentation of the loss of balance between Yin and Yang as shown in Figure 2 [5,9]. Shen is believed to reside in the Heart which is in charge of governing the Blood Vessels that need to be nourished by the Blood. When the Heart blood is deficient to fail to nourish the Shen, Shen is disturbed to lead to insomnia. Alternatively, Shen can be also disturbed by Heat in the body resulted from Blood deficiency. The relationship of insomnia and Blood deficiency is confirmed in the result of the study on the menopausal women aged 45 60 years with acupoints of Sanyinjiao (SP6), Shenmen (HT7), Baihui (GV20), Shenting (GV24), Yintang (M-HN-3), Guanyuan (CV4), Qihai (CV6), and Anmian (N-HN54) [10].

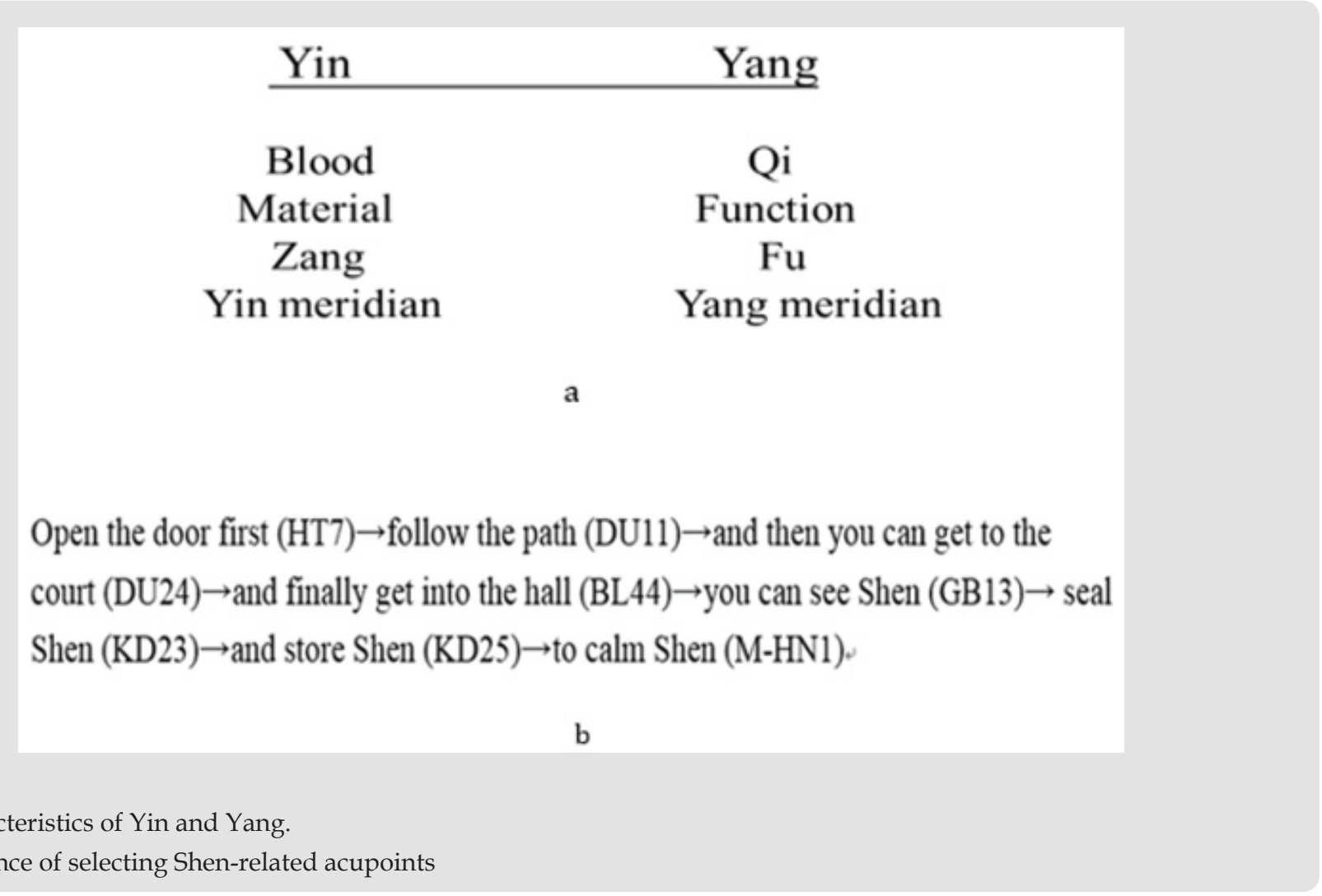

\section{Understanding Shen-Related Acupoints}

Chinese characters, different from the alphabet languages only representing forms and sounds, are called ideographs with three features in forms, sounds and meanings. It is argued that the language may be a barrier to hinder the TCM and acupuncture learners from acquiring the accurate meaning an acupoint refers to
[11]. In other words, understanding the meanings in Chinese of the acupoints may provide the better path to get the whole picture of Shen-related acupoints.In addition to HT7 (Shenmen), which has been often used in the most protocols as the main acupoint in the studies treating insomnia to effectively improve the quality of sleep [12-15], there are seven more Shen-related acupoints, which can 
be understood literally in Chinese as Table 1. Based on the theories of channel and Zang-Fu organs, the acupoints listed in Table 1 show that the Heart-Kidney represents the Yin-Yang, while KidneyBladder indicates the exterior-interior relationship.

Table 1: Characteristics of Shen-related acupoints [5].

\begin{tabular}{|c|c|c|}
\hline Acupoint & Meaning & Actions \\
\hline HT7神门 & Door to Shen & Regulates and tonifies the Heart to calm Shen \\
\hline BL44神堂 & Hall of Shen & Regulates Qi to activate the channel and alleviates pain to calm Shen \\
\hline KD23神封 & Seal of Shen & Lowers rebellious Lung Qi and Stomach Qi to calm Shen \\
\hline KD25神藏 & Storage of Shen & Eliminates Wind to calm with resolving phlegm \\
\hline GB13本神 & Root of Shen & Clears Heat and pacifies Wind to calm Shen \\
\hline DU11神道 & Path of Shen & Eliminates Wind to benefit the brain and calms the Shen \\
\hline DU24神庭 & Court of Shen & Eliminates Wind to calms the Shen \\
\hline M-HN1四神聰 & Calming Shen &
\end{tabular}

Blood is the key role in treating insomnia because Blood stasis occurs when Qi is impeded. Blood, which interacts with Qi viewed as the moving agent to maintain the circulation in body, is considered to be the nourishing agent in the TCM. It is discussed Qi is inconceivable without Blood and vice versa, which highlights the concept that Qi is the commander of Blood, while Blood is the mother of Qi. In addition, the circulation of Qi and Blood that must depend on the balance of Zang-Fu organs, such as Lung, Heart, Liver, Spleen, Kidney, and San Jiao explains the relationship that Blood can nourish Qi and Qi moves Blood [4]. In terms of the functions, Shen-related acupoints shown above seem to play the roles guiding practitioners with meanings in Chinese to establish the priority of use in practice. Judging from the Chinese meanings of acupoints, the practitioners are stongly advised to follow the sequence in Figure 2 to treat insomnia with the severity of insomnia.

\section{Discussion}

The meanings of acupoints in Figure 2 definitely explain the popularity of HT7 selected in the protocol to treat insomnia because an acupuncturist cannot treat insomnia at all without opening the door to the Shen. Acupuncture has been widely used for long and verified to reduce the symptoms of insomnia effectively [16]; however, it cannot be ignored that only the accurate selection of acupoints can play a crucial role for efficiency. HT7 selected along with the acupoints Baihui (DU-20), Shenting (DU-24), Benshen (GB-13), Sishencong (EX-HN1), Neiguan (PC6), Sanyinjiao (SP6) in the trial is verified crucial for the successful outcomes with its meaning in Chinese [3], which seems to imply that the functions of HT7 in the protocol treating insomnia indicate the language barrier deserves the attention clinically and academically [11]. On the other hand, HT7 also suggests the relationship between Prenatal Jing and Postnatal Jing needs attention. Long-term diseases with emotional issues can lead to disturbed Shen often presented with dull eyes. It is stated in TCM that the shining and bright eyes are the representations of the healthy state of Shen, showing the excellent Blood circulation and the strength of the Jing stored in Kidney and
Qi produced by Spleen. However, Shen cannot exist without Blood conveyed by Qi. Unfortunately, Qi is impaired when Jing, which can be converted into Blood, is damaged. In other words, Shen can be nourished as long as Jing and Qi are sufficient.

One of the functions of Kidney, which is classified as Water, is to store Prenatal Jing. The controlling sequence shows Water (Kidney) controls Fire (Heart), which indicates KD 23 and KD25 located on the Kidney channel can be selected to treat insomnia. In addition to the source of Blood converted from Prenatal Jin, Blood formation is simultaneous with the formation of Qi, explaining Blood is closely related to Qi in the TCM and can be formed from the Postnatal Jing derived from food and fluids. It deserves attention that Wind occurs to disturb Shen when Blood is deficient on account of Spleen qi deficiency. Governing Vessel (Du Mai) entering both Heart and the Brain to establish a pathway link with Shen is viewed as the sea of all the Yang channels. Digestion is a key function of Spleen to transform food into Blood and Qi and transport both to the four limbs. However, Spleen fails to perform the functions when Spleen qi deficiency indicating poor metabolism occurs. A long as Blood deficiency occurs, Wind arises in the body to interfere with sleep. In other words, tonififying DU11 and DU24 on the Governing Vessel can enable Wind to be dispersed to boost Spleen qi again.

\section{Conclusion}

Whether or not language can be a barrier for the TCM and acupuncture learners in the West remains uncertain. However, this study shows that each acupoint bears a specific meaning presenting the underlying functioins, which can enable learners and practitioners to explore the real actions. The concepts of Qi, Jing, and Shen in TCM and acupuncture theories are valuable criteria for diagnosis and treatments for insomnia since they are vital for human life. It deserves more research to link theses concepts with scientific evidence in the future, even though they are not convincing and cannot be explained and recognized accurately and properly by the biomedicine at present. 


\section{References}

1. Cao H, Pan X, Li H, Liu J (2009) Acupuncture for treatment of insomnia: a systematic review of randomized controlled trials. J Altern Complement Med 15(11): 1171-1186.

2. Ohayon MM (2002) Epidemiology of insomnia: what we know and what we still need to learn. Sleep Med Rev 6(2): 97-111.

3. Guo J, Huang W, Chu ying Tang, Gui Ling Wang, Fan Zhang, et al. (2016) Effect of acupuncture on sleep quality and hyperarousal state in patients with primary insomnia: Study protocol for a randomised controlled trial. BMJ Open.

4. Hong TZ (2019) Stagnated Liver-Qi-Based Insights into the Effects of Vinegar-baked Radix Bupleuri. Advancements Bioequiv Availab.

5. Hong TZ (2017) Exploring a New Extra Point for Sub-acute Cough: A Case Report. Scholar's Press, Germany.

6. Sacred Lotus Chinese Medicine.

7. Hong TZ (2018) Challenges in Learning and Understanding Traditional Chinese Medicine and Acupuncture. Open Acc J Comp \& Alt Med 1(1).

8. Hong TZ (2018) Reminders for Clinical Application of Extra Acupoints. Curr Trends Biomedical Eng \& Biosci 16(3).

\section{ISSN: 2574-1241}

DOI: 10.26717/BJSTR.2020.24.004037

Tong Zheng Hong. Biomed J Sci \& Tech Res

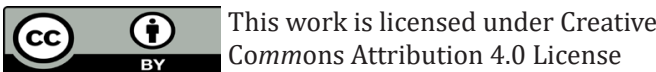

Submission Link: https://biomedres.us/submit-manuscript.php
9. Maciocia G (1989) The foundations of Chinese Medicine. Library of Congress Cataloging in Publication Data, New York, USA.

10. Li S, Yin P, Yin X, Anna Bogachko, Tingting Liang, et al. (2019) Effect of acupuncture on insomnia in menopausal women: a study protocol for a randomized controlled trial. Trials 20(1): 308.

11. Hong TZ (2018) Language Barrier before You in TCM and Acupuncture Learning. Advancements Bioequiv Availab 1(3).

12. ShergisJL, Ni X, Jackson ML, Zhang AL, Guo X, et al. (2016) A systematic review of acupuncture for sleep quality in people with insomnia. Complementary Therapies in Medicine 26: 11-20.

13. Zhu YH, YANG Cui Hua, HE Lin xi, QIAO Ling, CHENG Ci Song (2019) Effect of electroacupuncture at "Shenmen"(HT7) and "Sanyinjiao"(SP6) on energy metabolism in paraventricular nucleus of hypothalamus of insomnia rats. Zhen Ci Yan Jiu 44(3).

14. Cao Y, Yin X, Soto Aguilar F, Yiping Liu, Ping Yin, et al. (2016) Effect of acupuncture on insomnia following stroke: study protocol for a randomized controlled trial. Trials 17(1): 546.

15. Huang W, Kutner N, Bliwise DL (2009) A systematic review of the effects of acupuncture in treating insomnia. Sleep Med Rev 13(1): 73-104.

16. Huang W, Kutner N, Bliwise DL (2009) A systematic review of the effects of acupuncture in treating insomnia. Sleep Med Rev 13(1): 73-104.

\begin{tabular}{ll}
\hline BIOMEDICAL & Assets of Publishing with us \\
\hline RESEARCHES & - Global archiving of articles \\
\hline ISS: $2574-1241$ & - Immediate, unrestricted online access \\
\hline
\end{tabular}

